\title{
Tangence
}

\section{Le renvoi de la balle acadienne : 1953 , de France Daigle}

\section{René Plantier}

Numéro 58, octobre 1998

Le postmoderne acadien

URI : https://id.erudit.org/iderudit/025980ar

DOI : https://doi.org/10.7202/025980ar

Aller au sommaire du numéro

Éditeur(s)

Tangence

ISSN

0226-9554 (imprimé)

1710-0305 (numérique)

Découvrir la revue

Citer cet article

Plantier, R. (1998). Le renvoi de la balle acadienne : 1953 , de France Daigle.

Tangence, (58), 56-65. https://doi.org/10.7202/025980ar d'utilisation que vous pouvez consulter en ligne.

https://apropos.erudit.org/fr/usagers/politique-dutilisation/ 


\section{Le renvoi de la balle acadienne: 1953, de France Daigle}

\section{René Plantier}

Dans le préambule de son roman 1953, dont le sous-titre est Chronique d'une naissance annoncée ${ }^{1}$, France Daigle poursuit la création qu'elle définissait dans une étude de l'avant texte comme "la coexistence relativement pacifique de plusieurs niveaux de confusion " ${ }^{2}$; c'est là, selon l'auteure, que se situerait "le côté éventuellement moderne." Roman, chronique, documents, autobiographie cachée, analyse critique, références littéraires, historiques et médicales sont autant de forces contradictoires qui animent ici l'écriture. L'adverbe "éventuellement" ajoute à ces tensions la distance de l'humour qui peut devenir ironie. L'auteure cite Roland Barthes, avec gourmandise et avec un certain sourire. Nous citerons à notre tour les lignes suivantes:

langue et style sont des forces aveugles; l'écriture est un acte de solidarité historique. Langue et style sont des objets; l'écriture est une fonction: elle est le rapport entre la création et la société. ${ }^{3}$

La première ligne du roman-chronique est: "La balle revient. Chaque balle est un défi. "Cette phrase revient elle aussi en refrain et en énigme métaphorique au long du texte ${ }^{4}$, avec des variations de forme, suivant les contextes. Par exemple: et la balle revient ( $p$. 128) avant une analyse psychologique de Brigitte, dans une analogie filée:

Quelque chose colle à elle, quelque chose comme la balle qu'elle ne cesse de retourner, et qui ne cesse de se dédoubler... Brigitte ne sait pas de quelle façon elle est prise au jeu. À vrai dire, elle ne sait même pas de quel jeu il s'agit. (p. 128)

1 France Daigle, 1953. Chronique d'une naissance annoncée, Moncton, Éditions d'Acadie, 1995. Dorénavant, les renvois à cet ouvrages seront indiquées dans le texte, entre parenthèses.

2 André Gervais, N.B.J., 1986, p. 44.

3 Roland Barthes, Le degré zéro de l'écriture, Paris, Gonthier, 1953.

4 P. $10,12,66,67,68,128,129,143,160$. 
Les surprises de l'amour et du désir, sans oublier le livre à faire, sont dans la prise de balle: "au bord d'une prise ou d'une perte de conscience. Les mots à dire à Élizabeth ne viennent pas." (p. 129) La signification ouverte de la référence est encore plus riche dans le dernier exemple du livre, avec le déplacement qu'apporte la comparaison:

Quelque chose vacille. Elle voit pourtant la balle. Elle rebondit par ci, par là, mais sans venir dans sa direction. Comme si la balle s'était mise à jouer son propre jeu. Brigitte regarde encore. Attend. Attendra. Que la balle se décide. Que la balle revienne. Car chaque balle est un défi. (p. 160)

Toutes les références à ce "tennis" sont liées à Brigitte et à Élizabeth. Ce n'est pas une constatation anodine, l'amour, en dépit des apparences, en dépit de la place linguistique réduite qu'il occupe dans les cent soixante-cinq pages du livre, est bien la visée profonde et le secret de l'écriture comme de la survie. La balle, en mouvement, est ici linguistiquement située, mais elle est cachée dans les autres instances du livre. Nous nous proposons donc de mettre en lumière les autres partenaires du jeu et les autres manières de renvoyer la balle acadienne: d'une génération à l'autre, d'une institution à l'autre, d'une histoire datée à un avenir humain aléatoire.

Le roman-chronique est dédié au père et à la mère de l'auteure. Ce n'est pas une simple marque affective habituelle. 1953 est la date de naissance de l'auteure. Il s'agit à la fois d'une gestation qui aboutit à un être nouveau et à une œuvre nouvelle en Acadie. La création conduit à une différence, elle est aussi une continuité. Le père journaliste est un mainteneur de l'Acadie, il est un acteur dans l'écriture quotidienne en français. Il y a donc un hommage dans le livre, à travers l'institution d'un journal. Il y a aussi un retour de la balle à travers l'écriture d'un roman-chronique en Acadie, par la bonne nouvelle d'un enfant, aux prises avec les nouvelles du monde. L'image de la balle s'inscrit dans l'immensité de l'univers et dans l'exiguïté du terrain de jeu acadien. Le travailleur du langage éphémère engendre une actrice de la pérennité, même relative.

La mère de Bébé $M$. est à l'origine de son langage, elle est, en qualité de lectrice et de spectatrice, le relais entre l'écrivaine qu'elle porte et l'univers. De la même manière, Garde Vautour, lectrice de L'Évangéline, est le lien entre l'hôpital de Moncton et 
58

les événements d'Angleterre ou de Russie, entre la maladie de l'écrivaine: "Car un romancier ne vit pas, il broie" (p. 81), et sa survie par l'invention d'un nouveau rapport au monde. Les personnages du "scripteur engagé sur la nation acadienne" (p. 56), de la mère et de Garde Vautour sont les intercesseurs de Bébé $M$. et de l'Acadie, dans la marche universelle du temps. Ils sont les témoins actifs, humains et vulnérables, face aux grands simulacres: le couronnement de la Reine, les funérailles de Staline, et les plus petits: le sacre de $M^{g r}$ Leménager (beau nom, en vérité!) ou l'inauguration de l'immeuble de la Société mutuelle L'Assomption. Ils collaborent à l'écriture dans leurs pensées et leurs opinions sur les faits rapportés par L'Évangéline, tandis que "le scripteur engagé" offre les articles qu'il écrit sur les événements du monde ou sur "les petites nouvelles de paroisse" (p. 161).

Il faut ajouter à cette masse d'informations animée par les lecteurs, les ombres de l'ouvrage précédent écrit par l'auteure, non par souci d'intertextualité ou de la mise en abyme "si chère à la littérature postmoderne" (p. 30) mais par obsession de l'essentiel, c'est-à-dire "la vraie vie" ${ }^{5}$. Apparaissent donc par surprise Claude, le masseur et Élizabeth. Toutes les formes susceptibles de renvoyer la balle ayant été suggérées, il s'agit de dévoiler la variété des retours de balle.

La litote acadienne est notée par France Daigle, dans une étude parue dans De l'avant-texte, à propos de sa trilogie:

Je me suis trouvée à produire textuellement une caractéristique fondamentale de la psyché acadienne, soit une retenue, un silence, que côtoie par ailleurs une langue fort imagée. (p. 42)

Elle y fait aussi allusion dans 1953, à la page 44. La litote établit une connivence dans la discrétion des moyens, elle place la dignité dans une faiblesse apparente. Elle domine paradoxalement la situation d'une minorité, face à l'ostentation du spectacle et face à la colonisation des images collectives. La mort de la reine Marie est rapportée dans L'Évangéline:

Même le père de Bébé $M$. y alla d'un éditorial bref mais respectueux précisant qu'il aurait été déplacé de souligner, en cette occasion, la signification de la monarchie britannique pour le Canada français. (p. 35)

5 France Daigle, La vraie vie, Montréal, l'Hexagone, 1993. 
Le non-soulignement est bien plus chargé d'allusions qu'une affirmation agressive. Il ne sépare pas et donc il n'exclut pas. Lorsque France Daigle cite les lignes écrites par son père après ces mots rapportés, on s'aperçoit que les généralités cachent les aspects politiques et les aveux de la conscience acadienne, sous l'égide des grandes autorités traditionnelles, c'est-à-dire la Providence, l'Église catholique, sans parler de la modalisation dans le style: "La Providence semble avoir suscité vers le trône d'Angleterre, depuis plus d'un siècle, un élan de respect qu'il est difficile de nier" (ibid.). Le jeu des adverbes est aussi révélateur: "Et si l'on considère le respect qui aujourd'hui encore, est généralement consenti à la famille royale..." (p. 35) La phrase finale citée revient d'ailleurs à la modalisation par une emphase plutôt démocratique: "Dans notre monde bouleversé, ne semble-t-il pas que la dignité des rois est motif d'espoir dans le triomphe d'un ordre social où chacun joue dignement son rôle?" (p. 36)

Certes, certes, belle vision que voilà pour les rameurs acadiens dans la nef du Nouveau-Brunswick et belle interrogation dont la rhétorique est perverse.

De la même manière, la réaction des deux Acadiennes, en lisant que la Reine avait une préférence pour un de ses enfants, tisse un réseau de connotations: "Elle ne savaient pas si ce genre d'aveu était acceptable chez les protestants, ou s'il s'agissait pour la famille royale de rappeler qu'elle faisait encore partie malgré tout de la race humaine. " (p. 37)

L'adverbe repris et souligné par le "malgré tout", le tour en interrogative indirecte sont autant d'affirmations critiques déguisées. La distance acadienne ménage des surprises dans les clausules d'énumération:

Le fils préféré de la Reine pleura durant toute la cérémonie intime qui se déroula devant quatre reines, deux rois et quelques autres membres de "la noblesse dépérissante d'Europe.... (ibid.)

La dernière formule en italique renvoie à L'Évangéline. On a tout lieu d'évaluer avec précision la valeur de l'indéfini "autres" qui contamine discrètement les six nobles précédents.

Les faits qui se sont déroulés pendant l'année 1953 sont donc fortement liés à la conscience acadienne. L'impertinence de Garde Vautour et de la mère de Bébé $M$., même si elles n'ont pas 
60

lu Le degré zéro de l'écriture (p. 43), présente une intelligence, une mémoire et un souci de la vérité partagé par le journaliste de l'Évangéline "qui n'avait pas lu Le degré zéro de l'écriture non plus" (ibid.), et par Trotski et Staline! (p. 39) L'élévation du débat atteint son sommet lorsque L'Évangéline explique: "que la reine ne règne pas sur nous par droit divin, ni même par notre choix, qu'en fait nous participons de sa dignité sans la lui conférer." (p. 54)

Le distinguo s'accompagne d'un sourire matois et le renvoi de la balle est à la hauteur du défi, parce qu'il se fonde sur le contexte local. Si la litote ouvre le sens avec des signes discrets, le phénomène de l'emphase produit la distance exacte et soulignée d'un retour victorieux.

C'est dans la prolifération des métaphores et de l'hyperbole que la grandeur court les plus beaux risques. La mère de Bébé $M$. et Garde Vautour, sous la direction de l'auteure, émettent des jugements d'un bon sens modéré, à propos d'Édouard VIII. Elles manifestent une compréhension attendrie jusqu'au moment où l'emphase succède à la simple information:

l'abdication de leur fils énergique et charmant par amour pour une Américaine deux fois divorcée enfonçait cruellement le glaive de la réalité dans le portrait royal. (p. 50)

L'armement de l'abstrait, armement de style noble, conduit au jeu de massacre. L'hyperbole accompagne la moquerie: "Car il ne faut pas oublier que depuis un certain temps déjà, l'ensemble de la monarchie oscillait telle une immense pierre précieuse sur le pic de la désuétude. " (Ibid.)

La vision est ravissante, la comparaison, en style noble elle aussi, sombre dans une représentation incohérente. Cette technique du jeu qui consiste à lancer d'abord un langage non marqué, suivi d'un brusque changement de niveau de langue conduit au comique. En voici un autre exemple frappant:

Ainsi à 27 ans, épouse et mère de deux enfants, femme de maison, femme d'affaires et propriétaire, Élizabeth accepta de devenir à son tour "un support de l'axe sur lequel se meut l'univers. (p. 55)

Tout le langage employé pour le couronnement repose sur des métaphores codifiées. France Daigle rappelle malicieusement le chapitre "Écriture politique* du Degré zéro de l'écriture où 
Roland Barthes évoque "le drapé extravagant" (p. 41) du langage révolutionnaire, mais toujours relié à "un droit sanglant ou une justification morale." (p. 42)

La sacralisation du langage figé dans un rituel conduit ici à un fonctionnement abscons, tant il est passé par une sublimation de concepts et de siècles, sans parler de la domination chrétienne en Europe, en particulier. Les résonances politico-religieuses tournent à vide, ou presque, dans la laïcisation de cette Europe et dans la perte de son pouvoir sur d'autres continents. Le renvoi de la balle est cruel dans cette définition morte: "le sabre du souvenir" pour inciter la reine "à rendre justice" (p. 53), la reine comme "source de l'honneur", le roi et la reine "guidés par les étoiles visibles pour eux seuls" (p. 54). On peut y ajouter le globe, le sceptre et la croix. N'oublions pas non plus que ses poignets sont ornés des "bracelets de la sincérité " (Ibid.). L'accumulation des métaphores génitives endort la communication. L'archevêque officiant accomplit bien son rôle:

Dieu vous couronne d'une couronne de gloire et de droiture, afin que, possédant une foi juste et le fruit nombreux de bonnes ouvres, vous puissiez obtenir la couronne d'un royaume immortel par la grâce de Celui dont le royaume dure à jamais. (p. 53)

L'auteure, à ce moment, révèle que la mère de Bébé $M$. a le même âge que la reine! Ce qui entraîne un retrait ironique de l'emphase :

Elle ne s'enorgueillissait pas d'être reine du foyer pour autant. Elle n'avait pas conscience non plus d'avoir été l'objet de quelque faveur divine pour son rôle de mère catholique, "à la fois une infirmière, une prieure, une éducatrice, une martyre et une reine. (p. 56)

La formule entre guillemets est tirée d'un discours d'un prêtre américain, et elle apparaît dans le livre comme un écho plein d'ironie. (p. 25, p. 56, p. 99). La mère de Bébé $M$. célèbre plutôt le travail concret de la ménagère et, devant tout le savoir figé et mort qu'entraîne le couronnement, elle tire des conclusions réjouissantes : "elle aimait se laisser imprégner de ce savoir et apparentait l'expérience à l'effet d'une bonne tasse de thé." (p. 52)

Si l'Église participe hautement à la cérémonie royale, elle est aussi en elle-même une institution fondatrice de la société. Plus on avance dans le livre et plus l'Église est victime de la satire. 
D'abord l'attaque apparaît par bribes de langage: "l'esprit de sacrifice ", à propos des mauvaises copies des films français (p. 94), "pour l'humanité souffrante" (p. 84), en appelle au retour des infirmières. Les clichés et les stéréotypes religieux montrent un langage piégé qui ne véhicule qu'un sentiment d'aliénation. L'attitude clogmatique et autoritaire de l'Église méprise l'intelligence des personnes et leur liberté de jugement, elle s'appuie sur le passé en critiquant le cinéma, la presse et la radio:

Le cinéma [...] avait renversé les barrières dans l'enclos desquelles les hommes pouvaient penser conformément à leurs croyances ancestrales et leur mentalité nationale. (p. 90)

L'auteure souligne dans ses citations le caractère paradoxal de certaines formules qui suggèrent une religion du conformisme et de la passivité :

D'autres films, en donnant la recette d'un bonheur sentimental en marge de la morale ou fondé sur des valeurs matérielles, obligeaient les spectateurs à "lutter contre eux-mêmes pour vivre chrétiennement." (p. 91)

La balle acadienne rebondit sur les métaphores clichés en lectures équivoques, par exemple dans la phrase du cardinal Feltin, archevêque de Paris, "qui prophétisait que l'être humain ne pouvait pas "impunément "s'élargir la conscience." (ibid.)

La verve de France Daigle se fait voltairienne et il faut souligner les articulations de la pensée par les outils désinvoltes, "bref", "en somme", qui minent les prises de position. Les pages sur la censure du film The Moon Is Blue provoquent la même réaction. Le raisonnement de l'archevêque de Washington, cité en exemple, repose sur des arguments qui prennent la symbolique ou la Bible comme substituts de toute pensée critique:

Il vous appartient d'avoir assez de conviction pour s'abstenir d'y aller et prouver qu'un grand nombre d'entre nous trouvent que la lune est bleue parce que le bleu est la couleur de la Sainte Vierge à qui nous appliquons ces lignes du Cantique des Cantiques, "belle comme la lune, brillante comme les étoiles, terrible comme une armée déployée pour la bataille. (p.105)

Ainsi l'intolérance frappait les moyens intellectuels du haut clergé, au point que le film Martin Luther était jugé "nuisible et positivement mauvais... un danger moral et social". (p. 106) L'antiphrase domine alors l'écriture et le lecteur attentif ne peut que s'attacher à la vivacité de l'échange, comme les spectateurs 
du match de tennis qui apparaît dans L'inconnu du Nord Express. Il y a, en particulier, un usage corrosif des clichés anodins en apparence: "Le sacre de son Excellence $M^{\text {gr }}$ Leménager fut l'occasion pour l'Acadie moderne de 1953 de se manifester dans toute sa splendeur." (p. 112) Dans une gradation, avec un effet de cadence mineure soulignant, si l'on peut écrire, la chute, toute la biographie idéale du prélat affirmait que ce don d'un évêque "né sur son sol, nourri dans ses foyers, fortifié à l'ombre de ses églises, formé sous l'inspiration d'un passé qui se renouvelle dans ses gloires les plus pures, ressemblait à un coin du ciel tombé sur terre." (ibid.)

Nous n'évoquerons pas l'année mariale, ni la lettre pastorale publiée dans L'Évangéline, "une réclame de la compagnie Moncton Plumbing" occupant le reste de la page. Il y avait un écho particulièrement édifiant entre "les indulgences", les neuvaines etc. et l'invitation faite aux lectrices "d'électrifier leur vie avec les merveilles modernes". (p. 159) Quant au parallélisme qui existe "entre la clurée du quotidien L'Évangéline et celle du passage de Jésus Christ sur terre", il est préférable d'en savourer le mystère et l'à-propos.

D'une institution à une autre, la revendication de l'esprit critique et du libre examen se développe dans la variété des moyens linguistiques. La méfiance à l'égard du passé, présenté comme solution permanente à toutes les situations, substitut d'une pensée personnelle, construit le rythme d'une aventure et non d'une répétition. La royauté britannique et la royauté de l'Église sont des simulacres. En refusant toute fixation de sens, France Daigle, au-delà de l'ironie et de la satire, instaure le règne de l'humour ou de l'humeur, moyen de miner les forteresses. Alors les équivalences dévoilent un retour sur soi du sourire, comme expression d'une liberté à toute épreuve dans une parabole généralisée.

Par exemple, toutes les informations concernant Trieste, problème international, conduisent à établir une chaîne analogique désopilante:

Or il y avait dans le geste de la mère déposant Bébé $M$. dans les bras de Garde Vautour, quelque chose de semblable à la Yougoslavie cédant la zone portuaire de Trieste à l'Italie. La mère, comme la Yougoslavie, ressentait les aspects contradictoires de la reddition... De son côté, Garde Vautour, comme l'Italie, acceptait Bébé $M$. avec toute l'assurance du monde 
64

médical (c'est-à-dire le monde occidental) mais elle savait qu'il ne faudrait négliger aucun effort pour mettre Bébé $M$. (c'està-dire Trieste) sur la voie d'un développement sain. (p. 63)

Ainsi, les minorités et les petites nations donnent-elles des leçons de grandeur à l'Histoire! L'Acadie souriant de ses propres pesanteurs dépasse ses frontières et ses souvenirs. La modalisation vient encore au secours de l'humour, lorsque l'auteure rapporte les résultats de l'émission Singing Stars of Tomorrow, où les jeunes cantatrices acadiennes "s'imposèrent comme de dignes descendantes d'Anna Malenfant, dont la voix, semble-t-il, n'avait d'égal que les couchers de soleil en hiver et La petite poule d'eau de Gabrielle Roy" (p. 103). Cette désinvolture crée un voisinage explosif avec "Au cours de l'été, on avait annoncé que les taudis étaient de meilleure qualité au Canada qu'aux États Unis". (ibid.) Cette capacité de l'autodérision et de l'absurde incite le lecteur à se libérer lui-même de sa grandeur et de ses certitudes. Il peut alors savourer, avec toutes les précautions d'usage, la rencontre de la représentation du film Titanic avec "la première heure du jour du couronnement "(ibid.). Ainsi, il mesure la contingence dans l'universel, et réciproquement. Comme le démontrent la célébration des produits Barbour (p. 89) et celle, plus perverse encore, du Coke: "En général, le Coke était indiqué quand il y avait perte d'identité quelconque et qu'il était nécessaire de redevenir soi-même (tout travail demande une pause pour un Coke)." (p. 90)

L'ouvrage de France Daigle est bien une défense et illustration de l'identité. Quoi qu'il arrive, le calme et la lucidité souriante se saisissent des faits, qu'ils aient une dimension internationale ou qu'ils s'abîment dans le diocèse ou la paroisse, à Moncton ou à Moscou, aux U.S.A. ou aux îles Minquiers, objet d'une "querelle de 900 ans" entre l'Angleterre et la France (p. 83). Tous les obstacles sont dans le livre, mais le livre se fait, dans le passage du téléscripteur et du "scripteur engagé "à l'écrivaine. La masse des faits rassemblés, les citations nombreuses servent de tremplin à l'écriture et suscitent de multiples références à cette écriture: "Le romancier a non seulement le droit de fabuler, il en a même le devoir." (p. 61) La continuité du travail s'établit dans la ré-apparition des personnages de "la vraie vie". La chronique "broie" le temps et les nouvelles, elle les replonge dans le passé, alors que l'œuvre les dépasse. Les forces de l'esprit critique, de l'invention, de la mémoire créatrice déplacent les obstacles et 
appellent à la conscience personnelle, à la résistance aux "tentacules du rêve américain" (p. 84). C'est alors que les forces de l'humour inventent la juste distance par rapport à l'espace et qu'elles multiplient les chances des rencontres essentielles.

Ainsi la route qu'Élizabeth reprendra encore une fois vers Moncton, et vers la mer. Vers une sorte de bout du monde où elle se sent pourtant renaître. Mais avec quelque chose de plus définitif cette fois. De plus conscient. À un point tel que même son nom lui paraît moins étranger tout à coup. Élizabeth. (p. 136) 